\title{
Sensorial de suco de laranja com diferentes açúcares
}

\section{Orange juice sensory with different sugars}

\author{
Patrícia Inês Kemper Back ${ }^{1}$; Jocieli Steinhaus ${ }^{2}$; Maiara Figueiredo Ramires ${ }^{3}$; Douglas Stoll ${ }^{4}$; Marlon de \\ Castro Vasconcelos ${ }^{5}$; Fernanda Hart Weber ${ }^{6}$
}

${ }^{1}$ Acadêmica de Agronomia, Universidade Estadual do Rio Grande do Sul, Três Passos, 55-3522-2895, patrícia-back@uergs.edu.br. 2cadêmica de Agronomia, Universidade Estadual do Rio Grande do Sul, Três Passos, jocieli-steihaus@uergs.edu.br ${ }^{3}$ Acadêmica de Agronomia, Universidade Estadual do Rio Grande do Sul, Três Passos, maiara-ramires@uergs.edu.br; ${ }^{4}$ Acadêmica de Agronomia, Universidade Estadual do Rio Grande do Sul, Três Passos, douglas-stoll@uergs.edu.br; ${ }^{5}$ Doutor em Ecologia, Universidade Estadual do Rio Grande do Sul, Três Passos, marlon-vasconcelos@uergs.edu.br; ${ }^{6}$ Doutora em Tecnologia de Alimentos, Universidade Estadual do Rio Grande do Sul, Três Passos, fernanda-hart@uergs.edu.br.

\section{N O T A \\ C I E N T Í F I C A}

Recebido: 14/09/2018

Aprovado: 14/06/2019

\section{Palavras-chave: \\ Citrus \\ Açúcar mascavo \\ Mel \\ Teste de aceitação}

Key words:

Citrus

Brown sugar

Honey

Acceptance test

\section{R E S U M O}

O Brasil é o maior produtor e exportador mundial de suco de laranja. O suco de laranja é um produto complexo, formado por uma "mistura" aquosa de vários componentes orgânicos voláteis e instáveis, responsáveis pelo seu sabor e aroma, além de açúcares, ácidos, sais minerais, vitaminas e pigmentos. No entanto, devido ao sabor mais ácido característico do suco de laranja, muitos consumidores lançam mão do uso de açúcar cristal "comum" para o seu consumo, o que pode reduzir a qualidade nutricional do produto final. Dessa forma, objetivou-se avaliar a aceitação do suco de laranja adoçado com açúcar mascavo, mel, e açúcar refinado, avaliando a percepção sensorial dos consumidores. O trabalho foi desenvolvido na Universidade Estadual do Rio Grande do Sul. As laranjas da variedade Valência, após seleção, lavagem e sanitização foram submetidas ao processo de extração do suco manualmente, e depois de filtrado, foram coletados 4,5 litros de suco de laranja. Foram então, separados em três porções de 1,5 litros e diluídos com a adição de 0,5 litros de água e açúcar cristal "comum", açúcar mascavo e mel até atingir $14{ }^{\circ}$ Brix. O teste de aceitação foi realizado por uma equipe de 31 julgadores não treinados, e o suco de laranja foi avaliado em relação a sua aparência, sabor, acidez, doçura e impressão global, empregando para isso, uma ficha de avaliação com escala hedônica estruturada de nove pontos. O suco de laranja adoçado com açúcar refinado apresentou-se como a melhor opção dos avaliadores, apresentando notas mais altas em todos os atributos testados.

\section{A B S T R A C T}

Brazil is the world's largest producer and exporter of orange juice. Orange juice is a complex product, formed by an aqueous "blend" of various volatile and unstable organic components, responsible for its taste and aroma, as well as sugars, acids, minerals, vitamins and pigments. However, due to the more acidic flavor characteristic of orange juice, many consumers use the use of "common" crystal sugar for their consumption, which may reduce the nutritional quality of the final product. Thus, the objective was to evaluate the acceptance of orange juice sweetened with brown sugar, honey, and refined sugar, by assessing the sensory perception of consumers. The work was developed at the State University of Rio Grande do Sul. The oranges of the Valencia variety, after selection, washing and sanitization were submitted to the juice extraction process manually, and after filtration, 4.5 liters of orange juice were collected. They were then separated into three 1.5 liter portions in three 2 liter containers each, and diluted with the addition of 0.5 liters of water and "ordinary" crystal sugar, brown sugar and honey to $14^{\circ}$ Brix. The acceptance test was performed by a team of 31 untrained judges, and the orange juice was evaluated for its appearance, taste, acidity, sweetness and overall impression, using a structured hedonic scale evaluation form. Nine points. Orange juice sweetened with refined sugar was presented as the best option of the evaluators, presenting higher grades in all tested attributes.

\section{Revista Verde \\ ISSN 1981-8203 \\ Pombal, Paraíba, Brasil}

v. 14, n.3, jul.-set, p.480-484, 2019 doi: 10.18378/rvads.v14i3.6317 


\section{INTRODUÇÃO}

A produção comercial de citros cresceu em função da exportação do suco de laranja e ao longo dos anos, o Brasil se consolidou como o maior produtor mundial de laranja (FAO, 2017). Desde então, a laranja (Citrus sinensis) é a fruta mais cultivada dentro do grupo dos citros, representando cerca de $90 \%$ do total produzido no país (IBGE, 2017), sendo que o Brasil é responsável por cerca de $50 \%$ da produção mundial de suco de laranja e exporta $98 \%$ do que produz, alcançando assim $85 \%$ de participação no mercado mundial (ERPEN et al., 2018).

A produção brasileira de laranja está distribuída em todas as regiões do país, embora a maior parte esteja concentrada no Estado de São Paulo, juntamente com o Triângulo Mineiro, integrando o cinturão citrícola brasileiro, que é responsável por cerca de $80 \%$ da produção nacional de laranja. O estado de São Paulo produz cerca de 12,3 milhões de toneladas de laranja, o que representa $73 \%$ da produção nacional (IBGE, 2017).

A laranja é uma das frutas mais consumidas em todo o mundo, não só pelo seu bom gosto, mas também por seu valor nutricional, oferecendo uma combinação perfeita entre sabor e saúde. É uma fonte rica de ácido ascórbico (vitamina C), ácido fólico, vitamina B6, flavonoides, pectina e fibra alimentar. Contém também uma quantidade significativa de minerais como os sais potássio, cálcio, fósforo, magnésio e cobre (MARTINEZ, 2016). Além disso, o suco de laranja constitui um produto complexo, formado por uma "mistura" aquosa de vários componentes orgânicos voláteis e instáveis, responsáveis pelo seu sabor e aroma, além de açúcares, ácidos, sais minerais, vitaminas e pigmentos (CORRÊA NETO; FARIAS, 1999). Apresentando dessa forma, nutrientes suficientes para ser considerado um alimento saudável.

Outra característica importante é destacada por Silva et al., (2016), que considera bebidas de frutas como produtos promissores devido à tendência de aceitação em pesquisas de consumo, além de contribuírem para a redução de perdas póscolheita de frutos perecíveis.

Couto et al. (2018), avaliaram as características físicoquímicas para distinguir as cultivares de laranja, objetivando um melhor direcionamento para o mercado consumidor e/ou para o seu processamento.

O suco de laranja natural apresenta uma vida útil muito limitada, tornando o processamento térmico uma maneira de aumentar seu shelf life, porém deixando o produto com características sensoriais diferentes do suco natural "fresco" (CORRÊA NETO; FARIAS, 1999). No entanto, devido ao sabor mais ácido característico do suco de laranja natural "fresco", muitos consumidores lançam mão do uso de açúcar cristal "comum" para o seu consumo, o que pode acabar comprometendo a qualidade nutricional do produto final. Para substituir a doçura da sacarose, a legislação brasileira permite o uso de diferentes edulcorantes para produção de diversos alimentos, no entanto, cada um possui características específicas de intensidade e persistência do gosto doce e ainda presença ou não de gosto residual. E esses fatores são determinantes na aceitação de preferência e escolha por parte dos consumidores (CARDELLO et al., 2000).

Os testes sensoriais utilizam os órgãos dos sentidos humanos como "instrumentos" de medida e devem ser incluídos como garantia de qualidade de alimentos por ser uma medida multidimensional integrada e possuir importantes vantagens como, por exemplo, determinar a aceitação de um produto por parte dos consumidores (CARDELLO; CARDELLO, 1998) e, até mesmo para o desenvolvimento de novos produtos, reformulação dos produtos já existentes no mercado, estudo da vida de prateleira (shelf life), avaliação das diferenças e similaridades apresentadas entre produtos concorrentes, identificação da preferência dos consumidores por um determinado produto e, também, otimização e melhoria da qualidade (SGS, 2011).

Para medir a aceitação e a preferência dos consumidores com relação a um ou mais produtos, a escala hedônica estruturada de nove pontos é, provavelmente, o método afetivo mais utilizado devido à confiabilidade e à validade de seus resultados, bem como sua simplicidade em ser utilizada pelos provadores (VILLANUEVA et al., 2005).

Com isso, foram produzidos sucos de laranja adicionados de três diferentes tipos de açúcares (cristal, mascavo e mel) e esses produtos foram submetidos à avaliação sensorial com o objetivo de verificar a aceitação pelos provadores.

\section{MATERIAL E MÉTODOS}

O presente trabalho foi desenvolvido na Universidade Estadual do Rio Grande do Sul (UERGS), unidade em Três Passos (RS). As laranjas utilizadas no processamento do suco foram da variedade Valência (Citrus sinensis), adquiridas no comércio local do município de Três Passos-RS.

As laranjas após seleção pelo grau de amadurecimento, lavagem e sanitização com água clorada, foram submetidas ao processo de extração do suco com o uso de Extrator de Sucos. Foram coletados e filtrados 4,5 1 de suco de laranja. Logo após, foram divididas três porções de 1,5 Litro em três recipientes de plástico com volume de $2 \mathrm{~L}$ cada, onde foram diluídos com a adição de $0,5 \mathrm{~L}$ de água filtrada até completar o volume da garrafa plástica. Cada um dos sucos de laranja foi adoçado até atingir $14^{\circ}$ Brix com açúcar cristal "comum", açúcar mascavo e mel. As amostras de suco de laranja foram acondicionadas em garrafas pet de $2 \mathrm{~L}$ e conservadas em refrigerador $\left(\mathrm{T} \approx 12^{\circ} \mathrm{C}\right)$ até o momento da avaliação, cerca de 3 horas após a extração e preparo do suco.

As três formulações de suco de laranja foram avaliadas sensorialmente, na UERGS, por uma equipe de 31 julgadores composta por alunos, funcionários e professores de ambos os sexos, todos voluntários e sem treinamento.

Para a realização da análise sensorial foi adotado o método afetivo de escala hedônica estruturada de nove pontos e os atributos avaliados foram a aparência, sabor, acidez, doçura e impressão global. Com variação de 1 (desgostei extremamente) até 9 (gostei extremamente).

Os provadores receberam $40 \mathrm{~mL}$ de suco de laranja de forma monádica, na temperatura de $16^{\circ} \mathrm{C}$, em copos plásticos com capacidade volumétrica de $50 \mathrm{~mL}$, codificados com números aleatórios de três dígitos. Ainda, receberam um copo de água e bolacha salgada, para eliminar possíveis sabores residuais, entre a avalição de uma amostra e outra, além da ficha de avaliação.

$\mathrm{Na}$ interpretação dos resultados obtidos através da análise sensorial, pelo teste de aceitação do suco de laranja natural, foi realizado pelo o teste estatístico de Friedman, que é uma alternativa não paramétrica para o teste ANOVA de 
experimentos em blocos ao acaso (RBD - Randon Blocks Design), uma vez que as variáveis respostas são qualitativas e não possuem distribuição normal. Os dados das notas atribuídas foram utilizados com variáveis respostas e os avaliadores como bloco. O teste de Friedman é baseado em ranques e o teste post hoc, utilizado o algoritmo obtido apresentado por Galili (2010). As análises foram realizadas no ambiente estatístico R v.3.5. (The R Core Tean).

\section{RESULTADOS E DISCUSSÃO}

Os resultados obtidos para o atributo sensorial avaliado para o suco de laranja adoçado com açúcar cristal, açúcar mascavo e mel mostraram que há uma diferença na preferência (impressão global) do suco em relação à forma de adoçar o mesmo $\left(\chi^{2}=41.434, \mathrm{DF}=2 ; \mathrm{P}<0,001\right)$, o que pode ser observado na (Figura 1).

Figura 1. Notas dos sucos de laranja adoçados com açúcar cristal, açúcar mascavo e mel em relação a impressão global.

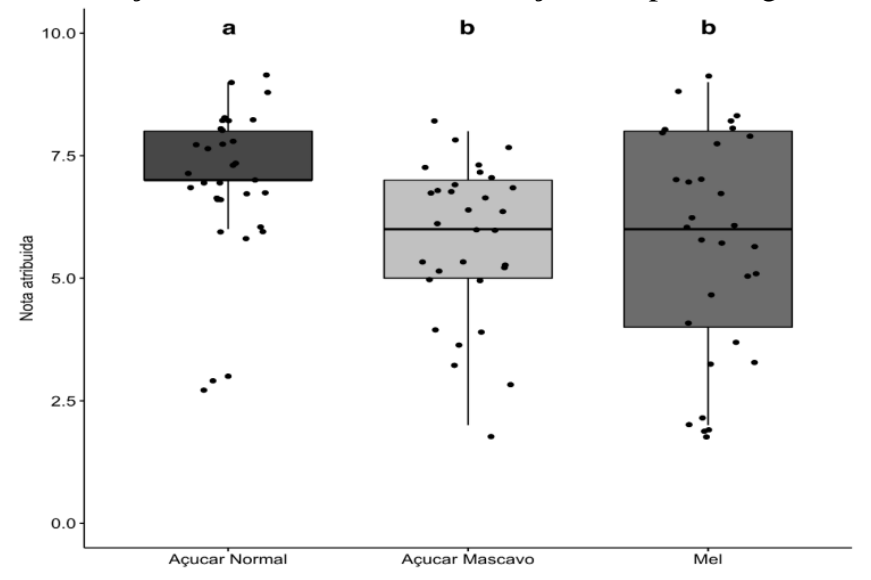

O suco de laranja adoçado com açúcar normal obteve maior aceitação pelos provadores, ficando com médias de 7 a 8 (gostei moderadamente/ gostei muito/ gostei extremamente), mostrando uma ótima aceitação do produto. O suco de laranja adoçado com açúcar mascavo foi o que apresentou menor aceitação pelos provadores, obtendo médias de 3 a 6 (desgostei moderadamente/ desgostei ligeiramente/ nem gostei nem desgostei/ gostei ligeiramente). E o suco de laranja adoçado com mel, obteve uma aceitação boa, ficando com médias de 5 a 8 (gostei ligeiramente/ gostei moderadamente/ gostei muito). Os três tipos de suco diferiram estatisticamente, ficando com as maiores notas o suco de laranja adoçado com açúcar cristal, seguido do suco adoçado com mel e depois o adoçado com açúcar mascavo.

Os resultados obtidos com relação a aparência estão apresentados na Figura 2. Com relação a este atributo sensorial avaliado, pode-se verificar que o suco de laranja adoçado com açúcar cristal obteve as notas mais altas, seguido das outras duas amostras, que por sua vez não diferiram estatisticamente.

Em estudo realizado por Leite et al. (2016) em que também foi avaliada a aceitação do suco de laranja com diferentes agentes adoçantes (açúcar, adoçante, stevia, demerara e stevia + adoçante) os autores verificaram que as amostras de suco de laranja adoçadas com stevia e demerara obtiveram os menores índices de aceitação em relação as outras amostras, pelo fato de não serem consumidos diariamente devido ao preço elevado, e sabor forte, mostrando-se inferiores se comparadas as demais.

Figura 2. Notas dos sucos de laranja adoçados com açúcar cristal, açúcar mascavo e mel em relação a aparência.

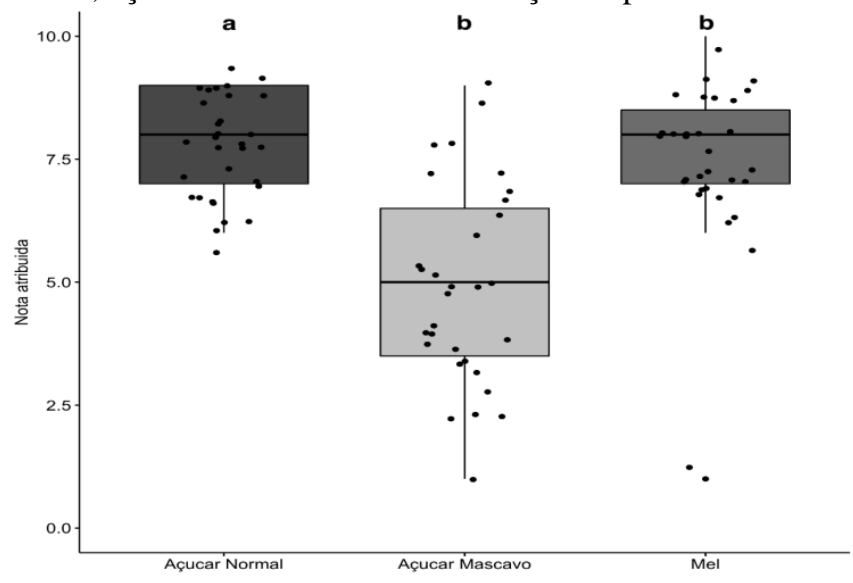

De acordo com Gava et al. (2008), muitos produtos alimentícios estão sendo modificados para uma formulação sem açúcar ou com opções mais saudáveis de carboidratos, que apresentem doçura similar à sacarose. $\mathrm{O}$ mel produzido a partir de abelhas Apis mellifera é um produto mundialmente conhecido, muito apreciado devido ao seu sabor e aroma, bem como pela sua qualidade nutricional (MANTILLA et al., 2012) e apresenta-se como uma alternativa mais saudável para adoçar os alimentos e sucos de frutas.

A avaliação para o atributo doçura está apresentada na Figura 3, onde pode-se verificar que não houve diferença significativa entre as amostras analisadas pelos provadores.

Figura 3. Notas dos sucos de laranja adoçados com açúcar cristal, açúcar mascavo e mel em relação a doçura.

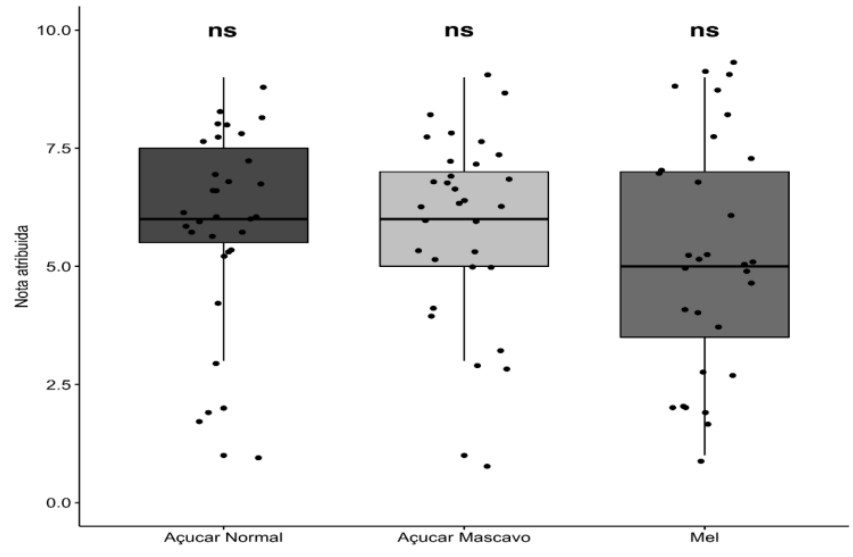

Para o atributo sensorial sabor, verificou-se que o suco de laranja com açúcar normal obteve as maiores notas, seguido do suco de laranja adoçado com açúcar mascavo. Ocorreu uma menor aprovação do sabor do suco de laranja adoçado com mel devido ao sabor residual característico deste carboidrato.

Silva et al. (2008), demonstraram que a elaboração de um néctar de caju adoçado com mel é viável dentro do processamento utilizado, tornando-se mais uma alternativa de aproveitamento e agregação de valor para o pedúnculo de caju e o mel de abelha em substituição a sacarose. Oliveira et al., 2018 verificaram que a redução gradual do açúcar em 
bebidas de frutas é uma estratégia viável que poderia contribuir para reduzir a ingestão de açúcar da população e, portanto, melhorar seu estado de saúde. Essas estratégias devem basear-se na percepção sensorial e hedônica do consumidor em relação à redução de açúcar. De acordo com os resultados deste trabalho, o açúcar pode ser reduzido em 5 a $8 \%$ sem afetar a percepção sensorial do consumidor, enquanto reduções na faixa de 10 a $20 \%$ podem ser implementadas sem alterações na reação hedônica do consumidor na avaliação de néctar de laranja.

A avaliação para o atributo acidez está apresentada na Figura 4, onde pode-se verificar que não houve diferença significativa entre as amostras analisadas pelos provadores.

Figura 4. Notas dos sucos de laranja adoçados com açúcar cristal, açúcar mascavo e mel em relação a acidez.

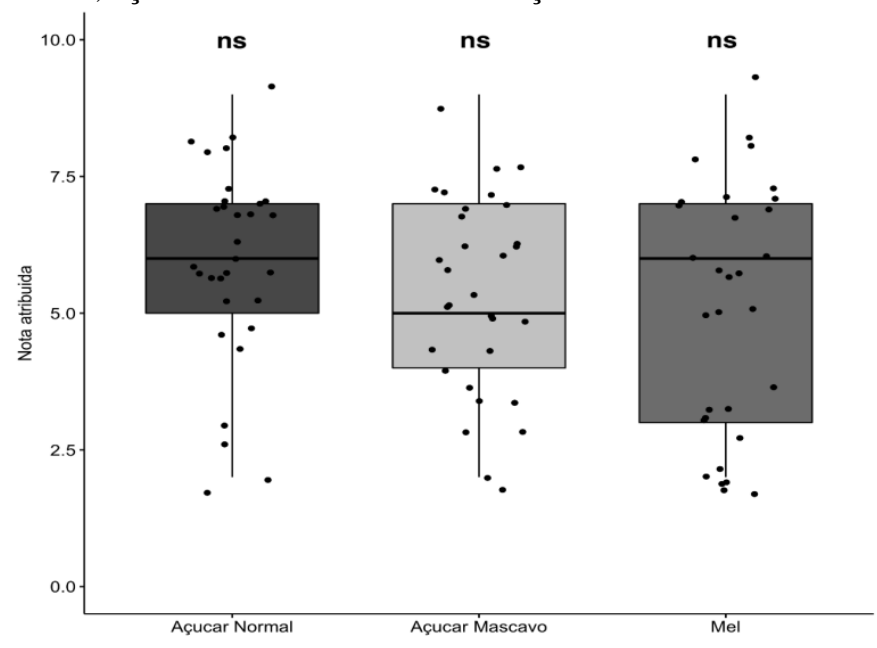

Com relação à cor, verificaram-se diferenças significativas entre as diferentes amostras. Para esse atributo, as menores médias foram obtidas com as amostras de suco adoçado com açúcar mascavo. Dessa forma, a rejeição ao açúcar mascavo pode ter ocorrido, por este apresentar uma coloração mais escura. Conforme Parazzi et al. (2009), o açúcar mascavo não passa por processo de cristalização ou refino, tendo como característica a sua produção, normalmente artesanal, para qual não são utilizados procedimentos de purificação do caldo, assim como a separação e secagem do açúcar, o que lhe confere uma coloração mais escura que vai dar a cor característica aos alimentos em que for adicionado.

Com os resultados das receitas avaliadas foi possível observar que assim como o sabor, acidez e doçura, a aparência é um atributo importante para a aceitação do suco de laranja pelos seus consumidores.

\section{CONCLUSÃO}

O suco de laranja adoçado com açúcar cristal é a melhor opção para o público, seguido do suco de laranja com mel e o suco de laranja com açúcar mascavo.

\section{AGRADECIMENTOS}

A Universidade Estadual do Rio Grande do Sul, Unidade em Três Passos, permitindo o desenvolvimento, fortalecendo pesquisas e implementação de trabalhos.

\section{REFERÊNCIAS}

CARDEllo, H. M. A. B; SILVA, A. A. P; DAMÁSIO, M. H. Análise Descritiva Quantitativa De Edulcorantes em Diferentes Concentrações. Ciência Tecnologia Alimentos, v. 20 n.3, p.15-18, 2000.

CARDELlO, H. M. A. B.; CARDELLO, L. Teor de vitamina $\mathrm{C}$, atividade de ascorbatooxidase e perfil sensorial de manga (Mangifera indica L.) var. harden, durante o amadurecimento. Ciênc. Tecnol. Aliment., v. 18, n. 2, p. 211 217, 1998. 10.1590/S0101-20611998000200013

COUTO, C. A.; SOUZA, E. R. B.; MORGADO, C. M. A.; OGATA, T.; JÚNIOR, L. C. C. Cultivares de Citrus Sinensis: alternativas para a diversificação de pomares brasileiros. Revista Brasileira de Fruticultura., v.40, n.5, e-097, 2018. $\underline{10.1590 / 0100-29452018097}$

CORRÊA NETO, R. S.; FARIAS, J. A. F. Fatores que influem na qualidade do suco de laranja. Ciência e Tecnologia de Alimentos, v. 19, n. 1, 1999. 10.1590/S010120611999000100028.

DE FARIAS SILVA, C. E.; DA GAMA, B. M. V.; OLIVEIRA, L. D. M.; DE ARAUJO, L. T.; DE ARAUJO, M. L.; DE OLIVEIRA JUNIOR, A. M.; ABUD, A. D. S. Uso da laranja Lima e seus resíduos no desenvolvimento de novos produtos. Revista Brasileira de Engenharia de Biossistemas, v. 10 , n. 1, p. 69-96, 2016. 10.18011/bioeng2016v10n1p69-96

ERPEN, L., MUNIZ, F. R., SOUZA MORAES, T., ROCHA T, E. C. Análise do cultivo da laranja no Estado de São Paulo de 2001 a 2015. Revista IPecege, v. 4, n. 1, p. 33-43, 2018. 10.22167/r.ipecege.2018.1.33

FAO. Food and Agriculture Organization of the United Nations 2017. Crops. Disponível em: <http://www.fao.org/3/a-i8092e.pdf $>$. Acesso em 10 jan. 2019.

GAVA, A. J.; SILVA, C. A. B.; FARIAS, J. R. G. Tecnologia de Alimentos: princípios e aplicações. São Paulo: Nobel, 2008.

IBGE, Instituto Brasileiro de Geografia e Estatística. Produção Agrícola Municipal - 2015. 2017. Disponível em: <https://sidra.ibge.gov.br/pesquisa/pam/tabelas >. Acesso em: 10 jan. 2019.

LEITE, I. F. S.; MOURA, T. C.; ALIXANDER, B. M. A.; PEREIRA, F. K.; FARIAS, F. G. Avaliação sensorial, microbiologia e físico-química de suco artificial de laranja adicionado de diferentes agentes adoçantes. Revista Verde de Agroecologia e Desenvolvimento Sustentável. v.11, n 4, p. 194-197, 2016. 10.18378/rvads.v11i4.4626

MANTILLA, S. P. S.; SANTOS, É. B.; BARROS, L.; FREITAS, M. Análise descritiva quantitativa aplicada em mel de abelhas (Apis mellifera): Uma revisão. Colloquium Agrariae, $\quad$ p. $75-84, \quad$ pp. 10.5747 .2012$. 10.5747/ca.2012.v08.n2.a081 
MARTINEZ, M. Laranja. Disponível em: <http://www.infoescola.com/frutas/laranja > Acesso em: $28 \mathrm{de}$ agosto de 2017.

OLIVEIRA, D., GALHARDO, J., ARESC, G., CUNHAB, L. M., DELIZAA, R. Sugar reduction in fruit nectars: Impact on consumers' sensory and hedonic perception. Food Research International. $\quad 107, \quad 371-377, \quad 2018$. 10.1016/j.foodres.2018.02.025.

PARAZZI, C.; JESUS, D.A.; LOPES, J.J. C.; VALSECHI, O. A. Análises microbiológicas do açúcar mascavo. Bioscience Journal, v. 25, p. 32-39, 2009.

PORTAL ACTION. Teste de Friedman. Disponível em: <http://www.portalaction.com.br/tecnicas-naoparametricas/teste-de-friedman>. Acessado em: 28 de Agosto de 2017.

R CORE TEAM. R: A language and environment for statistical computing. R Foundation for Statistical Computing, Vienna, Austria. 2019. Disponível em: <https://www.Rproject.org/>. Acessado em: 28 ago. 2018

SILVA, R. A., MAIA, G. A., DA COSTA, J. M. C., RODRIGUES, M. D. C. P., FONSECA, A. V. V., SOUSA, P. H. M., e CARVALHO, J. M. Néctar de caju adoçado com mel de abelha: desenvolvimento e estabilidade. Ciência e Tecnologia de Alimentos, Campinas, v. 28, n. 2, p. 348-354, 2008. 10.1590/1981-6723.18916

SGS do Brasil. Análise sensorial. São Paulo. Disponível em: 〈http://www.meuspa.com.br/download/AnaliseSensorial.pdf $>$ Acesso em 16 jan. 2019.

VILLANUEVA, N.D.M.; PETENATE, A.J.; DA SILVA, M.A.A.P. Perfomance of hibrid hedonic scale as compared to the traditional hedonic, self-adjusting and ranking scales. Food Quality and Preference, Oxford, v.16, n.8, p.691-703, 2005. 